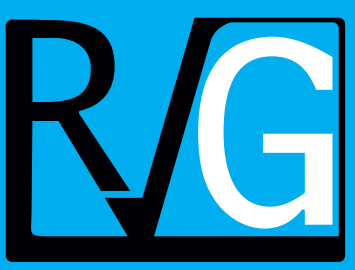

Año 21 No. 74

Abril - Junio 2016

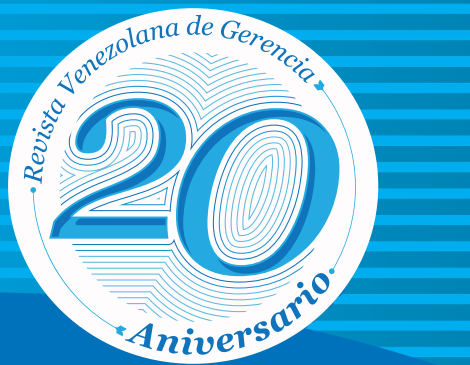

Venezolana de
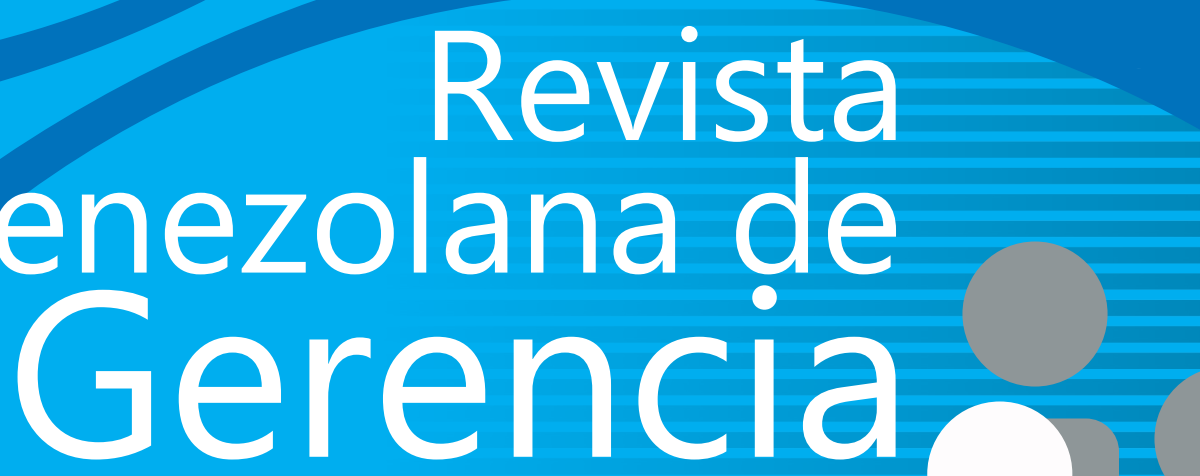


\title{
Revista Venezolana de Gerencia: Balance de dos décadas
}

\author{
Meleán Romero, Rosana ${ }^{1}$ \\ Marín Portillo, Kattiuska ${ }^{2}$
}

\section{Resumen}

En el presente artículo se caracteriza la trayectoria de la Revista Venezolana de Gerencia durante 20 años de gestión ininterrumpida, logrando precisar las principales fortalezas y debilidades de los procesos fundamentales de su gestión, así como los desafíos inherentes para los años venideros. Se revisan documentos y publicaciones que denotan la gestión editorial de revistas de alto impacto, siendo las vivencias en la revista un factor fundamental en los planteamientos y afirmaciones realizadas. Resaltan entre las fortalezas el posicionamiento y prestigio logrado, así como su presencia en redes sociocientíficas (repositorios, bibliotecas, catálogos y bases de datos) y la calidad certificada por reconocidos índices nacionales e internacionales. Su principal debilidad está representada por problemas presupuestarios, que limita el disponer de recursos necesarios para su funcionamiento. A veinte años de gestión, son muchos los logros alcanzados, acompañados de exigentes desafíos que orientan a seguir con pie firme, ofreciendo contribuciones de alto impacto que potencien la gerencia como área de conocimiento que de manera continua se construye y reconstruye para ofrecer soluciones asertivas a las complejas realidades que afligen al país y a la región latinoamericana.

Palabras clave: gestión editorial; proceso de edición; visibilidad; difusión; revista venezolana de gerencia.

Recibido: 15.05.16 Aceptado: 30.06.16

1 Docente-Investigadora del Centro de Estudios de la Empresa (CEE) de la Facultad de Ciencias Económicas y Sociales (FCES) de la Universidad del Zulia (LUZ). Directora de la Revista Venezolana de Gerencia (RVG). Estudiante del Doctorado de Ciencias Sociales, mención Gerencia de FCESLUZ. Acreditada en el Programa de Estímulo a la Innovación e Investigación (PEII), en el Nivel B (Convocatoria 2015). Correo electrónico: melean_rosana@fces.luz.edu.ve

2 Coordinadora de publicaciones de la Revista Venezolana de Gerencia (RVG) de FCES-LUZ, adscrita al CEE. Correo electrónico: kattymarin@yahoo,com 


\title{
Venezuelan Magazine of Management: two decades' balance
}

\begin{abstract}
In this essay is presented the Venezuelan Magazine of Management's characteristic trajectory, that its management had been uninterrupted for twenty years, achieving an extraordinary capacity in being precise when pointing out the principal strengths and weaknesses of its management's fundamental processes, as well as the inherent challenges for the upcoming years. Documents and publications, which denote the editorial's management for high impact magazines, are reviewed, being the experiences in the magazine a fundamental factor in the statements and assertions made. Among the strengths, the positioning and the prestige achieved stand out, as well as its presence in the social-scientific networks (just like repositories, libraries, catalogs and databases) and the quality certified by recognized national and international indexes. Its main weakness is represented as budgetary problems, which limit the availability of resources necessary for its operation. At twenty years of management, many achievements were accomplished, being accompanied by demanding challenges that guide to continue the trajectory with surefooted, offering high impact contributions that enhance management as a knowledge area, so that -in a continuous way - it is constructed and reconstructed for providing assertive solutions to complex realities that afflict the country and Latin America region.
\end{abstract}

Keywords: editorial management; editing process; visibility; diffusion; Venezuelan magazine of management.

\section{A manera de introducción}

Las revistas científicas, se constituyen en un medio de difusión del conocimiento generado en espacios de investigación, siendo a la vez la cara visible de instituciones educativas de alto nivel, potenciando su reconocimiento y prestigio tanto a nivel nacional como internacional. Como plantea Delgado et al, (2006), se han convertido en instituciones sociales que confieren prestigio y otorgan recompensas a quienes hacen posible su existencia.

Se constituyen en el principal medio de comunicación científica al que recurren autores y quienes consumen dichos conocimientos, para transmitir o responder a una necesidad informativa (Delgado et al, 2006; Osuna, 2006). Así, tanto investigadores como profesionales de disciplinas diversas, cuando quieren dar a conocer los resultados de sus investigaciones o inquirir acerca de nuevos descubrimientos, intercambiar experiencias o difundir información acerca de personas, instituciones o productos, acuden a las revistas científicas (Delgado et al, 2006).

Las revistas científicas responden a necesidades de áreas diversas del conocimiento. Desarrollan procesos de gestión que permiten obtener de manera periódica, productos científicos que enriquecen el conocimiento existente, potenciando sin duda su actualización constante. El reto, es que sean reconocidas por la calidad y el impacto de sus publicaciones a nivel de la comunidad científica mundial.

Para ello, deben asumir estrictos estándares de calidad, que parten por el cumplimiento exhaustivo de normativas, criterios y lineamientos durante el desarrollo de procesos clave de su gestión; de manera que estos se logren de forma eficiente, y por sobretodo apegados a preceptos de responsabilidad, honestidad y compromiso. 
Por un lado, se desarrollan procesos editoriales, cuyo resultado redunda en la obtención de las diferentes ediciones de la publicación como producto visible del trabajo previo realizado por un equipo de alto nivel. Este trabajo no solo incorpora actores internos a la organización a la cual pertenece la publicación; reúne colaboradores (evaluadores o árbitros) ${ }^{3}$ adscritos a organizaciones ubicadas en diversos contextos geográficos que comparten áreas de conocimientos comunes. Con una visión crítica, reflejada en los procesos de evaluación realizados, los evaluadores son quienes validan la pertinencia y calidad de cada trabajo y su potencial de publicación.

Como resultado del proceso de edición, se obtiene el número o edición específica de la publicación a ser distribuida, tanto de forma física, como digital e incluso empleando ambas modalidades. Inicia el proceso de distribución, a través del cual da a conocer el nuevo conocimiento científico generado. Su difusión debe garantizar la visibilidad y el acceso $0^{4}$ por parte de la comunidad científica general.

A partir de este proceso, se alimentan repositorios, bases de datos, bibliotecas e incluso Instituciones (Universidades, Institutos, Centros u otras unidades de investigación) y actores de la comunidad científica en general, garantizando accesibilidad al conocimiento publicado, el cual se constituye en la base para la conformación y consolidación de redes sociocientíficas por las que fluye el conocimiento, construido y reconstruido, de acuerdo a demandas y exigencias de entornos cambiantes, dinámicos y cada vez más exigentes.

Tanto el proceso de edición como el de distribución, deben ser ejecutados de manera conjunta garantizando calidad y eficiencia en aras de mantener la calidad y visibilidad de la producción científica generada.

En este sentido, se caracteriza la trayectoria de la Revista Venezolana de Gerencia durante 20 años de gestión ininterrumpida. Se describen y analizan aspectos clave de los procesos de edición y difusión y visibilidad, precisando debilidades y fortalezas, así como los principales desafíos asociados a la gestión editorial desarrollada. Se revisan documentos y publicaciones que permitan responder al objetivo trazado, a la vez que se recurre a información específica de la Revista Venezolana de Gerencia, y a experiencias derivadas de su gestión.

\section{Revista Venezolana de Gerencia: sus inicios}

En la década de los noventa se presencian en América Latina reformas orientadas a responder a presiones impuestas por la globalización económica. Estas reformas inciden

3 Nuestros árbitros, son actores que de manera directa y bajo un perfil ad-honorem desarrollas actividades de evaluación que sin duda contribuyen de manera directa con la calidad entregada en cada edición de nuestra publicación.

4 La Revista Venezolana de Gerencia, así como el resto de las Revistas de la Universidad del Zulia, se insertan en una política de acceso abierto, fundamentada en los principios que promueven el acceso libre a la información y el conocimiento científico, tecnológico, humanístico expresado en las declaraciones de Budapest (2002), Bethesda (2003) y Berlín (2002) (Universidad del Zulia - Rectoría, 2012). 
en el ámbito de la educación superior, propiciando dinámicas de crecimiento que exigen procesos de maduración de algunas tendencias, específicamente las asociadas con el crecimiento del nivel de postgrado y el desarrollo de proyectos estratégicos de ciencia y tecnología en países como Brasil, México, Argentina, Chile, Venezuela y Colombia (García, 2003).

Lo anterior exige el fortalecimiento del campo de la investigación, potenciando procesos fundamentales que permitan la generación de conocimiento de alto nivel en universidades latinoamericanas, resaltando entre ellas las venezolanas. Estos procesos, deben ir acompañados de actividades de difusión que den visibilidad al conocimiento generado en estos espacios de investigación.

Para incentivar este tipo de actividades de investigación, se crea la idea por decisión gubernamental en Venezuela, el Sistema de Promoción al Investigador (SPI) que acreditaría a científicos nacionales, a la vez que otorgaría bonificaciones para motivar y premiar a quienes rompiendo el esquema docentista tradicional entre otros factores adversos, produzcan conocimiento relevante (Eblen-Zajjur, 2001), según el autor, esto obligó a las universidades a incentivar y promover sus actividades científicas así como a incorporar y capacitar intensa y agresivamente recursos humanos que garanticen el incremento en el número de investigadores acreditados. Ante esta realidad, las universidades venezolanas comienzan a concentrar esfuerzos en esta materia, potenciando procesos y mecanismos para la generación y divulgación de conocimiento con pertinencia científica - social.

En la década de los noventa, las exigencias de las Universidades se orientan a la mayor divulgación de su actividad científica, pues, son diversas las críticas a las que están sujeta producto del poco debate para afrontar a la comprensión y solución de la crisis (Ochoa, 1996). De igual forma, plantea la autora que la creación del Programa de Promoción del Investigador (PPI) en el año 1990, demanda publicaciones de investigadores venezolanos.

La situación expuesta, aunada a la importancia asignada en ese entonces a la gerencia, cuya reforma era vital para la solución de la crisis de la época (Ochoa, 1996), son elementos que favorecen la creación de la Revista Venezolana de Gerencia. Es así como en junio de 1996, producto del esfuerzo de un equipo de trabajo altamente comprometido, con alto sentido de responsabilidad y gran trayectoria en el campo investigativo, nace esta publicación cuyo nombre designa la especialidad del conocimiento a difundir y su país de origen (Ochoa, 1996), remontándose sus inicios a una vieja aspiración de investigadores de La Universidad del Zulia, comprometidos con el desarrollo científico del conocimiento administrativo, hoy denominado gerencial, buscando llenar el vacío existente en nuestro país sobre la divulgación de temas sobre la gerencia (Ochoa, 1996), área de gran interés y pertinencia en la época que surge la Revista.

$\mathrm{Su}$ periodicidad inicial fue semestral; la cual se mantuvo hasta el año de 1998 cuando se comienza a editar de manera cuatrimestralmente, periodicidad que se extendió hasta el año 2000, dado que a partir de 2001 se comienza su edición trimestralmente (Ochoa, 2002), periodicidad que se mantiene en la actualidad (2016).

Sus inicios no fueron fáciles, así lo plantea su fundadora la Dra. Haydée Ochoa en diversas editoriales de la revista: el apoyo organizativo, el 
financiamiento e incluso la identificación del nombre de la revista, se constituyeron en puntos críticos superados paulatinamente, evitando el fracaso de la publicación en el corto plazo. Por el contrario, se asumió el compromiso con la calidad como principio fundamental en cada actividad y proceso desarrollado, lo que sin duda condujo al alto nivel en cada número publicado.

En palabras de Kliksberg (1998), colaborador e impulsador de la Revista desde sus inicios, acertadamente afirma que la Revista Venezolana de Gerencia “...es un ejemplo de cómo con recursos modestos, pero con metas bien inspiradas, compromiso y calidad profesional se pueden abrir espacios relevantes para la acción colectiva...", evidenciando el esfuerzo científico, serio y riguroso desde los primeros números editados; esto conllevó a ganarse el respeto e interés por los medios académicos de Venezuela y la región.

Desde sus inicios, la Revista Venezolana de Gerencia, tuvo un gran alcance internacional; participación de autores, árbitros e investigadores de diversos países con garantía de inclusión hacia bibliotecas, bases de datos, unidades de investigación y grupos de discusión clave en los cinco continentes (Bracho, 1997). Consolidando, según Córdova (1998), su contribución no solo al mundo de la investigación científica, sino también ofreciendo información sobre temas vigentes para la realidad nacional e internacional.
Estos inicios, fomentaron las bases que sustentan hoy día, el éxito de la revista, consolidada y reconocida tanto a nivel nacional e internacional. Su trayectoria, marcada con grandes logros, pero también con muchos desafíos, se constituye en la base para continuar su crecimiento. Así, luego de veinte años de trayectoria, en medio de fuertes vicisitudes e inserta en contextos cada vez más complejos y exigentes, tanto a nivel de conocimiento y soluciones, como a nivel de restricciones económicas, se debe seguir avanzando con la garantía de que la Revista Venezolana de Gerencia, será siempre para la Facultad de Ciencias Económicas y Sociales de la Universidad del Zulia un sello de calidad científica institucional.

A pesar de los acontecimientos por los cuales ha transitado, el sentido de pertenencia, el compromiso y la calidad profesional de quienes se han mantenido al frente de ella, ha hecho que este importante medio de divulgación se mantenga en el tiempo, alimentando periódicamente entornos académicocientíficos con contribuciones de alto nivel.

Hoy, sin un presupuesto que respalde la labor que realiza ${ }^{5}$, pero con ganas de seguir avanzando y creciendo, la Revista Venezolana de Gerencia, sigue asumiendo desafíos; en la lucha contra la adversidad e insertos en un contexto institucional cargado de carencias, seguiremos adelante, encaminando

5 En el año 2006, la Universidad del Zulia, atravesó una severa crisis financiera; sin embargo se realizaron serios esfuerzos para que esa situación no afecte publicaciones como la Revista Venezolana de Gerencia (Rincón, 2006), sin embargo, se sigue financiando. En el año 2014 aumentos en los presupuestos por el incremento de los costos de edición, impresión y distribución de la publicación, muestran la imposibilidad de cubrir dichas erogaciones con el presupuesto aprobado para las Revistas científicas de la Universidad del Zulia, correspondiente al año 2014 (Artigas, 2014), situación que obliga a mantener ediciones digitales de nuestras publicaciones a partir del año 2015, cuando finalmente se ve agotado el presupuesto correspondiente a la impresión de las revistas de la Universidad del Zulia, entre ellas la Revista Venezolana de Gerencia. Se asume el formato digital en nuestro caso a partir del segundo número del año 2015 , sin posibilidad de retomar la impresión, pues la situación presupuestaria de las Revistas se agudiza en el año 2016. 
esfuerzos, desde el Centro de Estudios de la Empresa, para que este sueño, de hace veinte años siga dando la batalla y cosechando éxitos en pro de nuestro academia y de una comunidad científica que confía en el trabajo de nuestra publicación.

\section{Gestión editorial de la Revista Venezolana de Gerencia: procesos clave}

La Revista Venezolana de Gerencia, al igual que otras publicaciones científicas, desarrolla procesos editoriales fundamentales que se han mantenido con el transcurrir de los años, garantizando su mejora continua y adaptación a los exigentes requerimientos de nuestros evaluadores y seguidores. Se responde, por un lado, a criterios de evaluación establecidos por índices, repositorios y otras instituciones certificadoras de la calidad de los productos entregados periódicamente, y por el otro, a la exigente demanda de conocimiento científico en el área gerencial por parte de nuestros seguidores.

En este sentido, se describen los procesos de edición y distribución desarrollados, precisando fortalezas y debilidades, como parte del diagnóstico realizado, de igual forma, los desafíos inherentes a cada uno de estos procesos.

\subsection{Proceso de edición}

El proceso de edición, se considera fundamental en la gestión editorial de cualquier publicación. En la
Revista Venezolana de Gerencia, este se inicia al recibir el artículo y culmina cuando se ha emitido el veredicto final de rechazo o publicación. Es un proceso continuo, que a pesar de ser rutinario en la fase inicial, está impregnado de calidad en etapas sucesivas. Permite certificar de manera inicial la pertinencia científica y social de la contribución recibida, así como su calidad aparente, en cuanto al cumplimiento de las normas establecidas.

Exige la verificación de datos vinculados con el autor que lo remite (filiación institucional), así como su carácter inédito, ambos necesarios para dar ingreso a la base de datos ${ }^{7}$. La contribución recibida, se asigna a un miembro del Comité editor para su revisión y evaluación; considerando la pertinencia entre el área en la que se inserta el trabajo y la especialización del evaluador. Este proceso se denomina revisión editorial y tiene como fin evaluar rigurosidad y pertinencia científico social del artículo, así como la correspondencia de este con las áreas de la revista. También se determina el aporte de la investigación al conocimiento objeto de estudio y los aspectos de forma relacionados con las normas de la revista $^{8}$.

Este proceso conlleva a una decisión inicial: 1) el artículo puede ser aceptado para someterse al proceso de evaluación por parte del equipo de árbitros; pero que 2) dependiendo del valor de la contribución, puede ser devuelto al autor para que incorpore elementos considerandos en la evaluación realizada por parte del Comité editorial; o 3) se rechaza por

6 En esta fase se asigna un código para la identificación posterior del artículo.

7 Estos criterios se encuentran definidos en el formato diseñado por la Revista para tal fin. 
el no cumplimiento de los criterios establecidos por la Revista. En cualquier caso, la decisión es comunicada de inmediato al autor.

Luego de recibir la versión corregida por el autor cuando corresponda (versión posterior de la revisión editorial), se verifican los cambios y en el caso de ser favorable se asignan árbitros, de lo contrario se rechaza por la no incorporación de lo solicitado.

La asignación de árbitros se apoya en una base de datos la Revista estructurada por áreas y/o en redes científicas; de ellas se seleccionan tres evaluadores ${ }^{8}$ (local, nacional e internacional), acreditados como investigadores activos y con experiencia en el área objeto de estudio; se envía la contribución, apegados al sistema doble ciego y estableciendo un tiempo prudencial de 21 día para la evaluación; sin embargo, en muchas oportunidades no se logran obtener las evaluaciones de los trabajos en este tiempo. Esto hace que esta fase de evaluación sea un punto crítico del proceso editorial en la RVG que debe mejorar su eficiencia en cuanto a tiempo de respuesta por parte de los evaluadores.

Cuando se excede el tiempo indicado, se realizan recordatorios o se decide cambiar los árbitros. Durante esta fase, es fundamental el compromiso de los evaluadores en aras de garantizar el cumplimiento de los tiempos y la calidad final del producto potencial a ser publicado; sin embargo, esta situación se complejiza cuando este trabajo se realiza "ad honorem" con cada vez menos incentivos intangibles.

Son los árbitros quienes con sus sugerencias aportan calidad en los trabajos a publicar, por ello deben velar por el cumplimiento de los criterios definidos por la Revista ${ }^{9}$ y dispuestos en el formato de evaluación y en las instrucciones para los árbitros. Tales criterios se apegan de manera exhaustiva a estándares de calidad nacionales e internacionales definidos por organismos evaluadores ${ }^{10}$. Estos criterios rigen la evaluación de las partes del artículo: título, resumen, palabras clave, introducción, desarrollo del trabajo, notas al pie de página, conclusiones, propuestas si las hubiera, tablas, cuadros y gráficos, referencias bibliográficas. Deben verificar que el titulo de cuenta del objeto de investigación y no del objetivo; que el resumen contenga los elementos clave establecidos (objetivo, metodología, resultados y conclusiones); que las palabras clave deriven del título y del resumen; que se presente una

8 Los árbitros son sugeridos por el coordinador editorial y es el comité editorial en pleno, o en su defecto el director quien aprueban su asignación.

9 Para mayor información sobre estos criterios visite http://produccioncientificaluz.org/index.php/ rvg/about/editorialPolicies\#custom-1, los mismo fueron establecidos durante la gestión de la Dra. Haydee Ochoa con apoyo directo de la Prof. Teresa Gamboa e Isabel Rodriguez, fundadoras de la Revista Venezolana de Gerencia

10 A nivel nacional, en el año 2004 se comienzan a asumir criterios establecidos por el Fondo Nacional de Ciencia Tecnología e Innovación (FONACIT), organismo nacional público que ejecuta financieramente las políticas estratégicas en materia de desarrollo de programas y proyectos del Ministerio del Poder Popular para Ciencia y Tecnología e Industrias Intermedias (MCTI) (Terán, 2011). En el año 2009, se indican cuarenta y ocho criterios denominados Términos de Referencia para la Acreditación y Financiamiento de las Publicaciones Científicas y Tecnológicas estos criterios (Terán, 2011) y en el año 2012 surge el ClaCaLIA. También se responde a criterios definidos por índices internacionales. 
adecuada justificación de la temática y/o el problema del conocimiento y/o de la realidad objeto de estudio en la introducción, explicando la metodología de forma clara y precisa ${ }^{11}$.

En el desarrollo las secciones deben presentarse de manera que permitan lograr el objetivo planteado, garantizando una organización interna coherente y equilibrada donde se evidencia el aporte del o los autores del artículo, para finalmente plantear conclusiones que manifiesten alto nivel de abstracción.

Recibidos los arbitrajes, se verifican y remiten al autor indicando el plazo de envío de la nueva versión. Esto dependerá del veredicto emitido por los evaluadores: 1) veredictos similares con ligeras modificaciones, se envía a corregir; 2 ) veredictos similares con tendencias sustanciales, ameritan una evaluación adicional, para tomar la decisión de enviar a corregir o negar el artículo, 3) si el veredicto es negado, se toma la decisión de rechazar la publicación del artículo. Todos los veredictos recibidos, son revisados por el comité editorial, quien avala argumentos establecidos por los árbitros y decide su envío a los autores, otorgando un tiempo prudencial para realizar los ajustes correspondientes, si es el caso.

Al recibir la versión post-arbitraje, esta es asignada a los miembros del comité editor para la verificación de los cambios incorporados en la nueva versión del artículo (se estima un plazo de una semana). Al evidenciar la mejora del artículo (cuando corresponda, pues esto dependerá de los veredictos recibidos), se procede a incluirlo en la programación en el número de la revista que considere el Comité Editor. En el caso de no evidenciarse la incorporación de los cambios sugeridos, puede considerarse, dependiendo del valor del trabajo, la devolución al autor, precisando los aspectos que deben ser incorporados (esta decisión aplicará sólo una vez luego de recibir la versión final del trabajo), de lo contrario, el artículo será rechazado. Posterior a este proceso, los artículos aptos para publicación, se editan y preparan para su montaje, diagramado y publicación final.

El proceso de edición, a pesar de estar claramente definido y presentar fortalezas que le han permitido certificar la calidad de la Revista, su prestigio y periodicidad, está expuesto a los diversos acontecimientos que se presentan en la realidad universitaria, afectada en muchos casos por conflictos laborales (paros, suspensión de jornadas de trabajo, cierre de los accesos al recinto universitario), problemas con la plataforma tecnológica (conexión a internet), problemas asociados con la infraestructura, por nombrar algunos. Estos inciden de manera directa en la ejecución del proceso, restando eficiencia $y$ afectando tiempos de respuestas. Por ello, se deben definir acciones para solventar estos momentos de crisis tanto para la institución como para la Revista, pues esto podría afectar su periodicidad, criterio de valor, que no debe interrumpirse.

Aunado a lo anterior, como otro elemento de interés y señal de alerta, es el descenso en la producción científica nacional, claramente reflejado en la RVG, cuyo número de artículos locales recibidos y publicados, ha 
ido en descenso en los últimos años. Lo anterior como consecuencia del escaso financiamiento a proyectos de investigación por parte del estado venezolano y de universidades públicas $^{12}$.

Esta situación se acentúa desde hace un par de años, cuando el Fondo Nacional de Ciencia, Tecnología e Innovación (FONACIT), canal institucional para el financiamiento de la ciencia, tecnología e innovación en Venezuela, no apertura convocatorias para el financiamiento de proyectos de investigación en humanidades y ciencias sociales $^{13}$ (Requena, 2008). Esto afecta la producción nacional, por proceder un alto porcentaje de artículos científicos de proyectos de investigación adscritos a instituciones de educación en diversas áreas del conocimiento.

Afirma, Aguado-López y BecerrilGarcía (2016) que existe una caída dramática de la producción científica venezolana, que habiendo registrado en 2009 sus índices máximos de artículos publicados, se comporta desde 2010 a la baja, llegando a 2014 con cifras de productividad menores que las que registró incluso en su momento más bajo: 2005; situación esta que evidencia la pérdida de liderazgo de Venezuela en los últimos años. Para el autor, esta caída impacta más severamente a las Ciencias Sociales y Humanas.

Es el conocimiento generado en las universidades venezolanas, la base científica que alimenta nuestras publicaciones. Al no existir una política pública que responda de manera efectiva a la realidad planteada, continuarán en descenso productos científicos de calidad en nuestro país, y sin duda en las ciencias sociales venezolanas. Para Osuna (2006) siendo las universidades los entes con mayor producción de conocimientos y de productos científicotecnológicos, al no recibir los recursos necesarios y suficientes para mantener niveles de excelencia en investigación, el sustento de las publicaciones científicas en las diferentes áreas continuará siendo pobre.

Se suma a la situación antes descrita, la falta de incentivos en el desarrollo de estos procesos editoriales. Presupuestos reducidos e insuficientes ${ }^{14}$ impiden la creación de planes de formación de manera constante, para mantener actualizado al personal en estas labores. Por ello, uno de los desafíos que se presenta en la RVG, es la conformación de un equipo de relevo entrenado y con alta competencias en gestión editorial.

La situación presupuestaria, también ha afectado labores de

12 El presupuesto de las universidades públicas en Venezuela es cada vez más deficitario (Hernández, 2016), lo que ha afectado de manera directa funciones de investigación, reduciendo, en el caso de la Universidad del Zulia, en los últimos años asignaciones por proyectos y cantidad de proyectos financiados.

${ }^{13}$ En cuanto a las investigaciones en ciencias sociales, paradójicamente, la Misión Ciencia, no contempla entre sus objetivos financiar proyectos en ese dominio del saber (Requena, 2008)

${ }^{14}$ El presupuesto asignado a la RVG correspondiente al año 2015, se agotó en la impresión de 50 ejemplares del primer número del año (Año $20, \mathrm{~N}^{\circ} 74$ ), no pudiendo imprimir las tres ediciones restantes de ese año $\left(N^{\circ} 75,76\right.$ y 77$)$. Todo ello en una situación de incertidumbre y preocupación por parte de editores e investigadores. A partir del año 2015, la Universidad emprende una política de publicación en formato digital para todas sus publicaciones. En el julio del año 2016, la Revista Venezolana de Gerencia, no ha recibido presupuesto alguno para respaldar labores editoriales. 
impresión en la Revista. En sus inicios, se imprimía en la editorial de la Universidad del Zulia, pero retrasos presentados en el montaje y diagramación, conllevaron a su impresión en talleres externos a la Universidad, impidiendo la situación presupuestaria de la universidad, continuar con este proceso a partir del año 2014, cuando el Consejo de Desarrollo Científico, Humanístico y Tecnológico (CONDES) ${ }^{15}$ retira apoyo financiero a las revistas, viéndose obligadas a migrar a formato digital. Con ello, se reducen costos de edición y de distribución.

Desde entonces el proceso editorial de las Revistas de la Universidad del Zulia, entre ellas la RVG, es asumido por el Sistema de Servicios Bibliotecarios y de Información de la Universidad del Zulia (Serbiluz), siendo su personal quien edita, diagrama y dispone en el repositorio institucional de Revistas científicas y humanísticas (Revicyhluz) nuestra publicación. A pesar del esfuerzo realizado, esta dependencia de la Universidad del Zulia, carece de personal suficiente para diagramar las 35 revistas de LUZ, por lo que se constituyen en un desafío importante para la RVG, asumir este proceso. Para ello, se requiere de equipos de computación adecuados con software especializados que permitan desempeñar estas labores, así como de personal preparado y capacitado. De esta forma, se ejercería total dominio de las labores editoriales por parte de nuestra Revista

En consecuencia, la falta o ausencia de presupuesto para las publicaciones, es el escenario propicio para idear mecanismos de autogestión presupuestaria, en los que se encaminen esfuerzos, con apoyo institucional para lograr financiamiento; bien sea a través de mecanismos de extensión como asesorías, tutorías, formación de personal externo, pago de contribución por parte de autores, entre otros. Con esto se apoyaría el desarrollo de labores editoriales (incentivos a árbitros) y otras asociadas a la promoción y divulgación del conocimiento científico generado por la Revista), tema de interés en un debate futuro.

La realidad planteada, conlleva a la adopción de desafíos por parte quienes gestionan revistas científicas en Venezuela. Se deben vencer obstáculos y continuar la marcha con pie firme, garantizando día a día la continuidad de las operaciones y la calidad de la publicación. El cuadro 1, resume los planteamientos antes esbozados, precisando la realidad actual de la RVG.

\subsection{Difusión y visibilidad}

La visibilidad y difusión de los productos académicos publicados en revistas científicas permite el acceso a estos por parte de la comunidad científica en general. Si no se realizan los esfuerzos respectivos en esta fase, el trabajo realizado durante el proceso de edición, quedaría sin sentido al no existir la posibilidad de consulta por parte de lectores y seguidores.

El proceso de difusión otorga visibilidad a la revista y es responsable de que el conocimiento científico generado llegue efectivamente a la comunidad científica, sea reconocido por su calidad y pueda ser referenciado en otras investigaciones. De no realizarse de manera adecuada, el conocimiento generado se mantendría oculto, sin

${ }^{15}$ El CONDES es el ente que financia la investigación en la Universidad del Zulia 


\section{Cuadro 1}

\section{Proceso editorial: Desafíos inherentes}

\begin{tabular}{|c|c|c|}
\hline Fortalezas & Debilidades & Desafíos \\
\hline $\begin{array}{l}\text { Posicionamiento de la Revista } \\
\text { a nivel nacional e internacional. } \\
\text { Prestigio de la publicación y de } \\
\text { la institución de adscripción. } \\
\text { Sujeto activo en redes socio } \\
\text { científicas. } \\
\text { Alianzas con instituciones de } \\
\text { investigaciones nacionales e } \\
\text { internacionales. } \\
\text { Talento humano con sentido de } \\
\text { pertenencia, responsabilidad y } \\
\text { compromiso. } \\
\text { - Periodicidad de la publicación. } \\
\text { - Artículos de calidad. } \\
\text { Presencia en repositorios y } \\
\text { base de datos nacionales e } \\
\text { internacionales. } \\
\text { Contribuciones inéditas y de } \\
\text { impacto en el área gerencial. } \\
\text { Base de datos de árbitros } \\
\text { nacionales e internacionales }\end{array}$ & 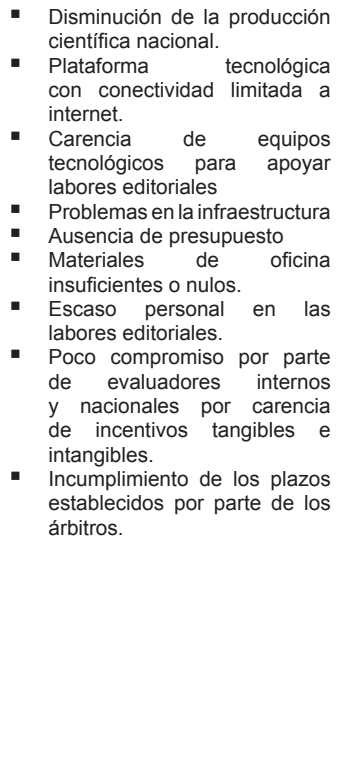 & 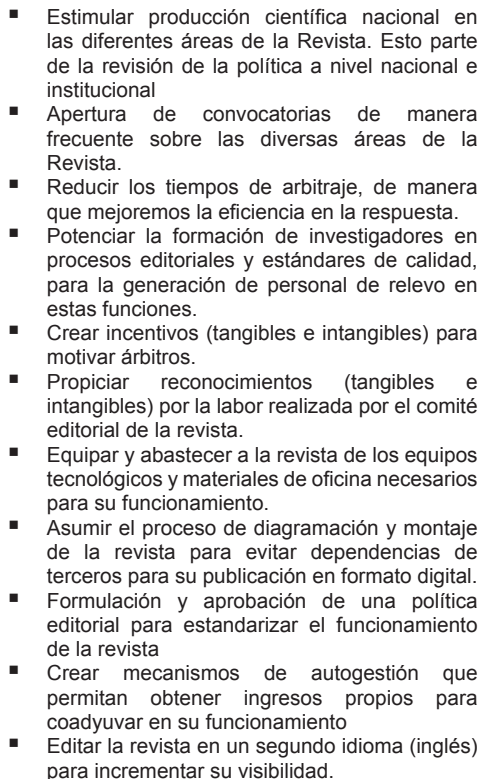 \\
\hline
\end{tabular}

Fuente: Elaboración propia

mayor impacto en las diferentes sociedades científico-sociales. Ochoa (2004:163), respalda lo anterior al plantear que la difusión del conocimiento tiene lugar cuando la producción intelectual llega al usuario, es decir, va más allá del trabajo de edición; lo hace visible al ponerlo a la vista del usuario potencial a fin de facilitar su consulta. Sin visibilidad no hay uso del conocimiento que es en definitiva la razón de ser de su producción.

A través de este proceso, las revistas proyectan sus productos y su calidad, incidiendo de manera directa en la relación de citas de la revistas y su consecuente factor de impacto ${ }^{16}$; este último, fundamental tanto para la publicación, como para articulistas, seguidores y colaboradores en general. Refiere Terán (2011), una revista no es buena solo por el hecho de que se difunda, sin embargo, es un criterio de visibilidad y accesibilidad que suma a la calidad de la publicación.

Para Ochoa (2004), la visibilidad de las revistas científicas puede lograrse a través de estrategias; estas

${ }^{16}$ Se debe trabajar por lograr un factor de Impacto alto; esto indica que los artículos publicados tienen una gran difusión y son citados numerosas veces por los autores cuando escriben un nuevo artículo (Miyahira, 2008). 
van desde las muy tradicionales, hasta el uso de los modernos recursos producto del desarrollo de la informática, ellas son: a) distribución del material impreso y electrónico (CD): Venta (en librerías y suscripciones), canje con otras publicaciones y donaciones; b) registro de la revista en bases de datos (directorios, catálogos e índices), a través de distintos medios de comunicación, hasta no hace mucho tiempo en materiales impresos, más recientemente mediante versiones electrónicas (CD e INTERNET, con tendencia al predominio de esta última); c) registro de la revista en lo que se ha denominado Hemeroteca Virtual; y d) creación de portales institucionales donde se alojan las revistas inmediatamente después de editadas.

La Revista Venezolana de Gerencia durante sus veinte años de trayectoria, ha transitado por todas ellas ${ }^{17}$, realizando hasta el primer trimestre del año 2015, la distribución de revistas impresas, respondiendo a convenios de canje, donaciones y suscripciones; sin embargo, producto de la migración a formato digital en este mismo año, se ha potenciado, su presencia bases de datos (directorios, catálogos e índices), como CLASE y LATINDEX, y hemerotecas virtuales (gratuitas) como Redalyc, entre otras, siendo su principal repositorio: Revicyhluz (Revistas Científicas y Humanísticas de la Universidad del Zulia), (produccioncientifica.luz.edu. ve), biblioteca digital de la Universidad del Zulia, lugar donde se reúne toda la producción científica de la institución.
Es de destacar que dada las limitaciones presupuestarias de la publicación, se recurre a bases de datos y hemerotecas y bibliotecas digitales gratuitas, donde se puedan difundir contenidos en acceso abierto. Según plantea Ochoa (2004), detrás de muchas bases de datos existen propósitos de rentabilidad y sólo se puede acceder a ellas a elevados costos, lo cual limita su consulta por la comunidad científica. Para la autora, ésta es una visibilidad que va acompañada de ciertos niveles de garantía de calidad de la revista, debido a que las bases de datos, especialmente los índices, evalúan la edición para incorporarla.

El hecho de que las publicaciones científicas se encuentren en las Bibliotecas y Centros de Documentación e Información facilita la búsqueda e identificación, la recuperación, la localización y la obtención de estos documentos y asegura el uso real de sus contenidos (Terán, 2011)

Sin embargo, este proceso se vio fuertemente afectado en años previos por problemas de conectividad asociados a la plataforma tecnológica, esto conllevo a cancelar un dominio externo para garantizar la visibilidad y accesibilidad de las publicaciones de la Universidad del Zulia. Altos costos acompañan esta decisión, sin embargo, son las Revistas científicas garantía de proyección institucional y posicionamiento de la Universidad que las respalda en el ámbito y ranking científico- académico.

Algunas fortalezas y debilidades se asocian con este proceso, y se precisan en el cuadro 2:

17 Para mayor información sobre este proceso y su ejecución consulte: Marín y Atencio (2008), Proceso Académico-Administrativo de la Revista Científica y Ética del Investigador: estudio de un caso. Revista Omnia, Año 14, No. 3. Disponible en: file:///C:/Users/usuario/Desktop/rvg\%20 katty\%20articulo\%20omnia.pdf 


\section{Cuadro 2}

\section{Difusión y visibilidad: Desafíos inherentes}

\begin{tabular}{|c|c|c|}
\hline Fortalezas & Debilidades & Desafíos* \\
\hline $\begin{array}{l}\text { - Versión electrónica gratuita } \\
\text { producto de la política de } \\
\text { acceso abierto en la que se } \\
\text { inserta. } \\
\text { Alianzas con instituciones } \\
\text { de investigación nacionales } \\
\text { e internacionales con } \\
\text { compromisos de canjes } \\
\text { intercambios y donaciones } \\
\text { Incorporación en bases } \\
\text { de datos, bibliotecas y } \\
\text { hemerotecas virtuales, } \\
\text { repositorios, catálogos. }\end{array}$ & 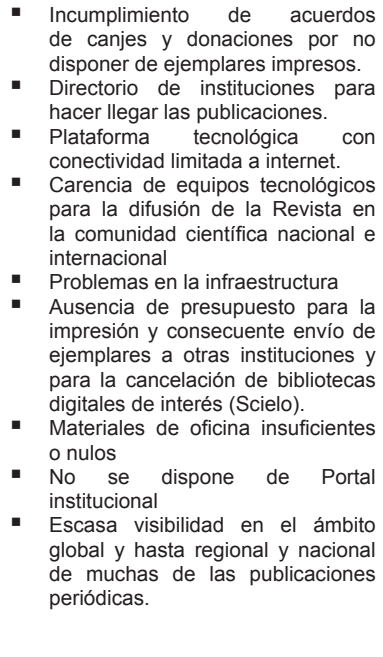 & $\begin{array}{l}\text { Fortalecimiento del canje electrónico } \\
\text { apoyados en el repositorio institucional de } \\
\text { la Universidad del Zulia (RevicyhLUZ) } \\
\text { Potenciar labores de difusión a nivel } \\
\text { nacional e internacional } \\
\text { Potenciar el incremento del número de } \\
\text { índices y repositorios que certifiquen la } \\
\text { calidad de la Revista y garanticen su } \\
\text { visibilidad. } \\
\text { Establecer nuevos convenios de } \\
\text { intercambio con otras comunidades } \\
\text { académicas e intelectuales del país y del } \\
\text { extranjero. } \\
\text { Incorporar al medio digital estadísticas } \\
\text { de uso que permita reflejar en qué grado } \\
\text { se alcanza el propósito de llegar a los } \\
\text { destinatarios de las revistas y su visibilidad } \\
\text { en el mundo. } \\
\text { Incluir un enlace a algún sistema de } \\
\text { traducción. } \\
\text { Actualizar datos referentes a aspectos } \\
\text { formales en el medio digital. } \\
\text { Establecer una normativa para el diseño } \\
\text { de las revistas científicas-electrónicas de } \\
\text { la Universidad del Zulia, con base de los } \\
\text { criterios de calidad exigidos en el ámbito } \\
\text { internacional. }\end{array}$ \\
\hline
\end{tabular}

*La definición de algunos desafíos se apoya en Díaz y Ortíz (2014) y Ochoa (2004)

Fuente: Elaboración propia

Para finalizar, se debe trabajar por potenciar la visibilidad y difusión de nuestra revista, logrando con ello incrementar el factor de impacto y la mejora en el posicionamiento actual de la publicación. Superar las restricciones y trabajar por mantener la RVG en posiciones importantes y de prestigio tanto a nivel nacional como internacional. Se dispone de calidad en el proceso editorial, y la garantía en la calidad de los trabajos publicados, más sino se dan a conocer, todos los esfuerzos previos carecerían de sentido. Actualmente se encaminan esfuerzos para la conformación de la Red de Revistas Científicas Venezolana, como un sistema cooperativo e interactivo que permita difundir el conocimiento científico generado con la garantía de visibilidad, accesibilidad e impacto científico-social.

\section{Reflexiones finales}

La Revista Venezolana de Gerencia ha llegado a sus veinte años de actividad ininterrumpida; transitando por escenarios adversos en los que ha tenido que ir venciendo obstáculos para no desvanecerse y desaparecer. Por el contrario, las vicisitudes atravesadas, la han hecho fuerte, encontrando en cada día de trabajo nuevas experiencias que le hacen crecer para seguir ocupando posiciones de prestigio a nivel nacional e internacional.

A veinte años de trayectoria, es reconocida como una de las principales 
revistas en el área gerencial, ganándose el respeto de reconocidos académicos e instituciones, no solo en su país de origen, sino también en países hermanos de la región latinoamericana, e incluso de otros contextos geográficos.

Con solidas fortalezas, y debilidades puntuales, asume los desafíos presentes para seguirá librando la batalla, en un contexto que depara amenazas acompañadas de muchas oportunidades por aprovechar. Con una gran labor por delante, seguirá en el debate científico, tributando la gerencia como área del conocimiento que se construye y reconstruye día tras día para aportar soluciones a las complejas realidades que afligen al país y a la región latinoamericana.

Agradeciendo a cada uno de sus colaboradores incondicionales, seguidores y especialmente a su equipo fundador y editores siguientes, por su constancia, dedicación y por emprender cada día un trabajo impregnado de calidad que ha logrado mantener la imagen de la Revista Venezolana de Gerencia, y de la institución que la respalda, en lugares de prestigio y respeto durante veinte años, y de seguro por muchos años más.

Para los que siguen en este transitar, nuestras palabras de estímulo para seguir ofreciendo, contribuciones de alto impacto, que sin duda enriquecen el conocimiento científico en el área gerencial. Asumir los retos y desafíos inherentes a la gestión editorial, es una lucha continua que se debe vencer día tras día.

\section{Referencias bibliográficas}

Aguado-López, Eduardo y Becerril-García, Arianna (2016), Producción científica venezolana: apuntes sobre su pérdida de liderazgo en la región latinoamericana, Revista Venezolana de Gerencia, Vol. 21, No.73, eneromarzo, Universidad del Zulia, Maracaibo, Venezuela. Disponible en: http://www.produccioncientifica. luz.edu.ve/index.php/rvg/article/ view/21054, Fecha de consulta: 20/04/16 pp 11-29

Artigas, Wileidys (2014), Desafío presupuestario para las revistas científicas de la Universidad del Zulia (Editorial), Revista Venezolana de Gerencia, Vol. 19, No.65, enero-marzo, Universidad del Zulia, Maracaibo, Venezuela.

Bracho, Domingo (1997), Editorial Revista venezolana de Gerencia. Revista Venezolana de Gerencia, Vol. 2, No.4, diciembre, Universidad del Zulia, Maracaibo, Venezuela.

Córdova, Edgar (1998), Editorial Revista venezolana de Gerencia. Revista Venezolana de Gerencia, Vol.32, No.6, diciembre, Universidad del Zulia, Maracaibo, Venezuela.

Delgado, E. Ruiz, R. y Jiménez, E. (2006). La edición de revistas científicas. Directrices, criterios y modelos de evaluación. (Texto en línea) Disponible en: http://recyt.fecyt.es/documentos/ Fecyt.pdf (Consultado: 2012, febrero 20).

Díaz, Mayra y Ortiz de Recasens, Zulma (2014). Indicadores de calidad para la visibilidad de las revistas científicas electrónicas de la Universidad del Zulia. Enl@ce. Revista venezolana de Información, Tecnología y Conocimiento, 11 (2), pp 91-111

Eblen-Zajjur, Antonio (2001), La Universidad Venezolana: ¿Docencia contra Producción Científica?, Acta Científica Venezolana, 52: 1-2, Facultad de Ciencias de la Salud Universidad de Carabobo, Venezuela.

García Guadilla, Carmen (2003), Balance de la década de los '90 y reflexiones sobre las nuevas fuerzas de cambio en la educación superior, en: "Las Universidades en América Latina: ¿reformadas o alteradas? La cosmética del poder financiero" Marcela Mollis (Compiladora). Colección Grupos de Trabajo de CLACSO. 
Hernández Fernández, Lissette (2016), Presupuesto universitario deficitario y la vigencia de la revistas científicas... Revista de Ciencias Sociales, Vol. XXII, No. 1, Enero-Marzo 2016, pp. 7-8. Universidad del Zulia, Maracaibo, Venezuela.

Kliksberg, Bernardo (1998), Editorial Revista venezolana de Gerencia, Revista Venezolana de Gerencia, Vol. 3, No.5, junio, Universidad del Zulia, Maracaibo, Venezuela.

Marín y Atencio (2008), Proceso AcadémicoAdministrativo de la Revista Científica y Ética del Investigador: estudio de un caso. Revista Omnia, Año 14, No. 3. Disponible en: file:///C:/Users/usuario/ Desktop/rvg\%20katty\%20articulo\%20 omnia.pdf

Miyahira Arakaki, Juan M. (2008). Criterios de calidad de las revistas científicas (editorial). Revista Medica Herediana 19 (1), Disponible en: www.scielo.org. pe/pdf/rmh/v19n1/v19n1ed1.pdf Fecha de consulta: 14 de febrero de 2016

Ochoa Henríquez, Haydée (1996), Editorial Revista Venezolana de Gerencia. Revista Venezolana de Gerencia, Vol. 1, No. 1 .

Ochoa Henríquez, Haydée (2002), Editorial. Haciendo historia: Origen de la Revista Venezolana de Gerencia. Revista Venezolana de Gerencia, vol. 7, núm. 20, octubre-diciembre, pp. 503506, Universidad del Zulia, Maracaibo, Venezuela.
Ochoa Henríquez, Haydée (2004), Visibilidad: El reto de las Revistas Científicas Latinoamericanas Opción, vol. 20, núm. 43, abril, pp. 162-168 Universidad del Zulia Maracaibo, Venezuela

Osuna C, Jesús Alfonso (2006), Conocimiento científico en nuestro país: Realidades y retos. Revista Venezolana de Endocrinología y Metabolismo, 4 (1), Febrero, Mérida, Venezuela. Fecha de consulta: 1 de marzo de 2016, disponible en: http://www.scielo.org.ve/scielo. php?script $=$ sci_arttext $\&$ pid $=$ S1690 $31102006000100001 \&$ Ing=es\&tlng=es.

Pérez Terán, Josefa (2011), Las revistas científicas y su visibilidad, Sapiens, Revista Universitaria de Investigación, vol. 12, núm. 1, enero-junio, Universidad Pedagógica Experimental Libertador, Caracas, Venezuela

Requena, Jaime (2008), Las investigaciones en ciencias sociales en Venezuela. Interciencia 33 (4), 242. Fecha de consulta: 01 de marzo de 2016. Disponible en: http://www.scielo.org.ve/scielo. php?script $=$ sci arttext\&pid=S0378$18442008000400001 \&$ Ing=es\&tIng=es.

Rincón, María Teresa (2006), Editorial Revista venezolana de Gerencia. Revista Venezolana de Gerencia, Vol. 8, No.21, enero-marzo, Universidad del Zulia, Maracaibo, Venezuela. 


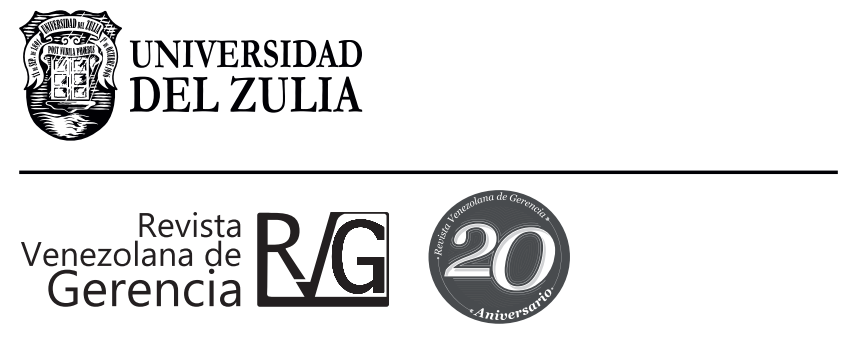

Año 21, No. 74

Esta revista fue editada en formato digital y publicada en junio del 2016, por la Universidad del Zulia, Vicerrectorado Académico, Serbiluz - Fondo editorial, Maracaibo -Venezuela. 